Having observed and reflected upon these facts about wounds and studied the theories on opsonins and the wonderful practical application of them by Sir Almroth E. Wright, $I$ argued thus. If so much depends upon the blood serum, why not try to increase the amount of it in and about healing parts by employing animal blood serum as the fluid with which to sluice, to irrigate, and to dress wounds? Pure healthy blood serum would seem to be ideal for this purpose, as it is essentially non-irritating and, in fact, has a soothing, cooling effect on injured parts. It is alkaline in reaction and from albuminous matters held in solution has a slight viscosity that gives it to a considerable degree air-excluding, and to a lesser degree adhesive, covering and protective properties which are all conducive to healing. It is now admitted by all pathologists, too, that normal blood serum possesses restraining influences on bacterial growth because of the antibodies it contains, and $\operatorname{Sir}$ A. E. Wright's researches have proved that blond serum also contains those substances which have the marvellous power of preparing microorganisms for ingestion by the white blood corpusclesviz., opsonins.

Here, then, in normal blood serum we have, I think, the best substance and the natural substance for perfect and rapid repair of wounds. The chief objection to even mild antiseptics has ever been that they have always some degree of irritative and devitalising effect on the tissues and there is no certainty that micro-organisms are de-troyed by their action. Indeed, there is little doubt but that the beneficial action of some antiseptics, and probably also of some aseptics, such as normal saline solution, consists in the fact that they do irritate the tissue and in this way ensure an increased outflow of serous fluid and stimulate the circulation about the healing parts. To supply the blood serum without applying the irritant ought to be better. But the researches of Sargent and Dudgeon have shown that wounds healing by first intention are not bacteria free, and it is quite possible that certain micro-organisms may be necessary to perfect union. Therefore, such a substance as blood serum, which inhibits but does not arrest all bacterial growth, would seem to be the most suitable.

For the purpose of putting these views to the test I obtained a quantity of fresh blood from the butcher, which had been poured directly from the incised aorta of a young, fat, healthy, two-year-old sheep into sterilised vessels. The blood was kept in air-tight glass jars for two days and, coagulation having taken place, the blood serum was then drawn off and secured in smaller sterilised glass air-tight jars. I am still doubtful how long to leave the serum in contact with the blood-clot, but the longer they remain together the darker the serum becomes, which is doubtless due to the hæmolytic action of the serum on the red blood corpuscles. I think what I have found to be the best serum for my purpose has been that which $\Upsilon$ syphoned away from the clearer collection at the top of a tall cylindrical vessel. I now have a supply of fresh blood every three days, and remove the serum every other day into the smaller, more portable, glass jars which I carry with me to operations. If the success of others employing blood serum is as great as in the cases in which $I$ have employed it, it may be that in the future normal serum may prove as useful and be as commonly placed ready to hand in our operating theatres as normal saline solution is at present.

It is mostly in ear cases that I have used blood serum. It seems particularly well suited for such, as there is so much bone excavation which leaves a dry as distinguished from a weeping wound. Desiccation in a wound is generally inimical to ready repair and this has been successfully avoided when blood-serum dressing has been employed. In every instance the soft structure healed by first intention. There was a singular absence of pain all through the time of recovery and the epidermisation in the ear cases seemed to proceed more quickly than usual.

Welbeck-street, w.

British Medical Temperance Association.The annual general meeting of this association will be held in the rooms of the Royal Medical and Chirurgical Society, 20, Hanover-square, London, W., on Tuesday, May $7 \mathrm{th}$, at $4 \mathrm{P}$ M. Tea and coffee will be provided at 4.30 P.M. At 5 P.M. a meeting will be held, open to all medical practitioners and students on presentation of visiting card, at which Professor G. Sims Woodhead, president of the association, will give an address on the Medical Justification of Total Abstinence.

\section{THE BALNEOLOGICAL TREATMENT OF URINARY DISEASES.}

\section{B Y DR. OS CAR KRAUS.}

(Concluded from p. 1153.)

I WILL now deal with the question, when can Carlsbad treatment promise a success and when ought surgery to assert its rights. The answer is not difficult. The progress in Roentgen technique has now made it generally possible, except in cases of very obese persons, to locate even small stones, especially if a compression-cylinder be used. The largest calculi that we ever saw passed through the natural passages in an attack of renal colic were of about the size of an almond (they are still in our possession). These were passed by a woman. Whether still larger stones have by any means a favourable chance of traversing the urinary passages without stoppage is questionable. At any rate, in the case of a man we should consider an operation indicated as soon as this size seems from the reduced radiogram to be considerably exceeded, or if the general symptom complex seems to demand removal of the stone. If the Roentgen examination be negative, or if the calculi be no larger than the size given above, it is proper to try the Carlsbad method. This treatment is by all means indicated after an operative procedure, in order to flush out the smallest particles, which can never be completely removed at a nephrotomy-particles like the gall-sand at a gall-bladder operation. The treatment is indicated after operation for the additional purpose of combating the catarrh of the urinary passages, especially that of the renal pelvis, and thus to guard against a new stone formation around the secreted mucus as a nucleus. Moreover, the influence of the basic water in the urinary acidity, when the case is of the acid variety, is to be desired. But even where this factor is not present, as in cystin stones, we may often note the useful effect of the mechanical washing-out of the particles. I well remember a patient several years ago on whom Zuckerkandl had done a nephrotomy; she carne from the sanatorium directly to Carlsbad and there passed large quantities of cystin sand and made a quick recovery.

We now come to the discussion of a class of patients who are continually being sent to Carlsbad-namely, those with stone in the bladder. Such patients ought to be sent to a mineral spring after a lithotripsy or a section has been already performed, and as soon after as they can be moved. Nowhere else will they so quickly lose the cystitis which is usually present, nowhere will they recover so rapidly, as under the bygienic régime and in the open-air treatment of a watering-place of this kind. The stimulation of urinary secretion and the regulation of the digestion never work so quickly and so beneficently as in these cases. Such patients are the most grateful that we see at Carlsbad, whether the concretion was uratic or phosphatic. I have in every such case observed a very considerable elimination of sandurate, phosphate, oxalate, or even cystin sand. I must emphasise again that this is not only and not always a case of purely chemical processes but of mechanical and biological as well, processes which, of course, I need not here discuss. I can say but one thing with certainty: urates are not "dissolved" by Carlsbad water. Small stones are swept out and the formation of new calculi prevented by the increase in metabolism. Large stones must be removed surgically, whether from the renal pelvis or from the bladder. Patients should be informed on this point, so that they will not demand the impossible. Occasionally, patients come to Carlsbad without the advice of physician in the hope of avoiding a lithotripsy. That is, of course, foolish. The stones, as we have said, cannot be dissolved. But when the stones are moveable, such a one may become impacted as a result of the long walks or of the railway journey itself. The same is true of kidney stones. I shall never forget a case of a young man who was sent to Carlsbad a few days after an attack of renal colic and before the expulsion of the stone. An hour after the departure from Vienna the stone left the bladder, but not being able to pass the orifice of the urethra it remained there until evening. I need not dilate on the sufferings of the poor man during the eight-hour journey. On his arrival I had to do a meatotomy in order to extract the almost almond-sized concretion with the forceps. Therefore one should always await the passage 
of the stone after an attack of colic before a journey is allowed.

Here also belongs the case of an old man who came to Carlsbad with a calculous cysto-pyelitis. He was very wretched and debilitated, with persisting fever and hæmorrhages, and his progress toward recovery was very slow. When the cystitis had somewhat improved he passea, as he had repeatedly done before, 15 or 20 calculi, either at home or in the course of walks, and quite without difficulty or pain. These were characteristic stones from the pelvis, either perfectly round or slightly faceted. In spite of this his slight symptoms, chiefly some strangury and frequency of micturition, would not yield. Cystoscopy showed the presence of a small stone in the bladder, which I removed by means of the aspirator. It would be worth while to recommend that after every attack of renal colic the patient should be cystoscoped as soon as the slightest indication arouses the suspicion that the stone was not passed. It net not be pointed out how well these small concretions are qualified to form the nucleus of a vesical stone, particularly when, as in this case, the folds of a vessie à porte.feuille tend to imprison the stone. Occasionally patients with a stone are sent to a watering-place to be healed of their cystitis before operation. That is, of course, so much pains wasted, for as long as the stone is there the fons et origo mali is not removed and the bladder cannot be healed. After operation, however, the case is quite different. As soon as patients can be moved they ought to "flush themselves out" diligently and lose their cystitis if such be present. By stimulating the peristalsis and loosening the bowels the circulatory disturbances in the pelvis are relieved; by increasing diaphoresis (with baths, \&c) and diuresis the toxins are eliminated and "urinary" fever is most successfully combated.

In this connexion we come to a condition about which a few remarks are called for. This is the so-called urio acid diathesis-an affection which seems to have increased prodigiously in recent years. Exactly what, then, is this uric acid diathesis? I have taken the trouble to make inquiries on this point among a number of excellent physicians and surgeons for the simple reason that $I$ have myself never seen a morbid type which would answer to this title, and I saw from their answers that they also were rather at a loss to make a definite statement. We often see the report in our uranalyses of a "heavy uric acid sediment" or "increased uric acid in the sediment," and this in the case of a patient who has nothing wrong with him but for a few more or less imaginary complaints. The conditions governing the solubility or insolubility of certain normal urinary constituents, such as the urates, the phosphates, uric acid, are not known certainly. Why is uric acid sometimes precipitated from solutions and sometimes not? And why the phosphates? How does it happen in one and the same individual that the taking of carbonated drinks at one time causes a cloudy or milky urine and at other times not? We are only too far as yet from understanding these most intimate

1 Latterly, it has become rather the fashion to undertake stonecrushing operations at watering-places. I am of the opinion that this is not advisable, since such a patient should not be asked to take a long journey. Nowadays, when surgical practice is no longer a prerogative of the few elect but has become fairly geueral there can be fourd in every large city, and in many of moderate size, men who can perform such an operation faultlessly and under the best of external conditions, in good nursing homes. The patients need not be carried far, and we do not see any reason why patients should need to be in a hospital or doctor's home at a watering-place, if they can get no benefit from balneological treatment, even of the best. Allow me to speak at this point of curious "fashion" which has lately appeared among patients with concretions-namely, that of going every year or two years to a water cure "to have their stone, which has formed again, crusbed." There are, as a matter of fact, people whose excretion of urates or phosphates is so rapid that relapses do occur-after a long period, rarely after short one. But when we hear that certain operators perform crushing operations regularly every year or two for a considerable number of their patients. we are moved to recommend them that they heed Guyon's advice and "carry the crushing to its utmost limits," and further, on no account neglect the "verifying examination" (Clinical Lectures, German edition by Oscar Kraus and Otto Zuckerkandl, vol. iii., pp 326 and 372 ). If this were done their cases might show a considerably diminished number of relapses. We know that genitourinary surgeons of prominence share our view. That it is reasonable is perfectly evideut. Only small fragments can go through the aspirator or be spontaneously passed. For this reason the old Bigelow method has been losing favour, because in this the crushing is made of less importance than the evacuation. The surgeon who wishes not to leave fragments behind to cause relapses will crush the stone to the smallest possible atoms. Bladder irrigation and stimulation of diure-is will wash out the last purticles, and it is just here Carlsbad may assert her rights in the after-treatment of a litho'apaxy. metabolic processes, processes which are certainly not purely or wholly brought about by chemical conditions. To be honest with ourselves we must admit that we know little more about the conditions under which uric acid is precipitated from the urine than that it happens regularly in the cold and also in circumstances which we are not able to explain satisfactorily. On this point the most trustworthy investigators are united; reference may be made among others to a piece of work by Ritter. ${ }^{2}$ Uric acid precipitates for unknown reasons from the urine of sufferers from arthritis or other diseases, but it also often does the same in that of healthy individuals, without any increase in the total acidity or in the content of uric acid. Moreover, the uric acid often precipitates without our being in a position even to draw any conclusions from the fact. We know, in short, as little about this as we do abont oxaluria and phosphaturia or about the alternation of oxaluria with uraturia or of phosphaturia with uraturia in the same person. All explanations which have been offered are unable to stand the test of criticism. We do know, however, that it is impossible to speak of an absolute or even of a relative increase in uric acid excretion without a complete examination into the metabolism based on comparative data for the same individual, and especially without taking account of the diet. To speak of "increased uric acid" in the sediment is nonsensical because we do not know the conditions of uric acid precipitation in health, or, in other words, what the "normal" amount of uric acid in the sediment is. However, what the apothecary or chemist reports as uric acid is usually amorphous sodium urate, oftem mixed with a few small whetstone-shaped uric-acid crystals. Practical experience has taught us that this appearance has no significance whatever, either clinically or pathologically; on this point I know that $I$ am in agreement with clinicians of note. One of these, Dunin, to whom we owe, among many other excellent researches, that paper on the relation between habitual constipation and neurasthenia, made this very question the subject of an investigation and comes to conclusions similar to ours. Unfortunately, the paper was written in Polish and is thus only accessible in reviews. ${ }^{3}$ The principal theses are the following: 1. The existence of an arthritic diathesis after the meaning of the French authors must be emphatically denied. 2. It is not proved that metabolism is at all slowed in diseases of this category. 3. From the superficial uranalyses as usually made in practice far-reaching conclusions, and especially diagnoses of "arthritism," are quite untrustworthy. 4. The supposition of a uric acid diathesis seems, in view of present knowledge, to be unwarrantable. 5. The significance of uric acid in the etiology of arthritis and of renal calculus is to be regarded at present as unknown. ${ }^{4}$ Among the many cases. which we have been able to follow with care during the last 18 years, we have found none where increased uric acid in the sediment had any meaning. On the other hand, we have again and again found for years before the appearance of calculi those characteristic large dumb-bells and the pointed forms of uric acid crystals to which Ultzmann in his lectures attributed a premonitory significance. We have always remembered particularly two cases where we achieved some renown as a prophet by cherishing this remark of Ult $z=$ mann. ${ }^{5}$ But these are only the premonitory signs of gravel.

There are no objections to be made on principle to the introduction of synonymous but relevant new terms, although the clearness of the conception rarely gains thereby. We know that the coronary asthma of to-day, the stenocardial attack of yesterday, and the angina pectoris of our fathers are one and the same. And so I have myself here and there made use of the expression "uric-acid diathesis" because it

\section{Zeitschrift für Biologie, Band xxv, No. 17}

4 Cited from Ziegler-Kahlden's Centralblatt, March 28th 1901

5 I have spoken of one of these cases in another publication (Report of the Meeting of the Paris Académie de Medecine and Annales ôes Maladies des Organes Génito-urinaires: La Pollaquiurie Lrique, cation before the Academie de Médecine the patient has been oblioed toron a small stone crule de Medecine the patient has been obliged. to bave a small stone crushed. The other case was of a man who had been sent to Carlstad on account of enlarged liver. I advised a change in his manner of living and threatened him with renal colic and resica stone. Both made their axpearance : first the colic, and two s ears latermentioned pointed uric acid blader myself. In both cases the abor mentioned pointed uric acid crystals had sbown themselves lor year nately exercise due to an ankylosis of the hip afrer a dret, and the lack oa the femur. 
was the fashion. But I should like to propose dropping it for «good and all, as we have dropped the "herpetic diathesis." They simply do not exist. But even if we do not recognise a uric-acid diathesis we do know the arthritis urica and gravel. Both lead to a deposition of urates in the organism. I described a symptom ten years ago in a communication to the Paris Académie de Médecine, ${ }^{6}$ which I called "pollakiuria urica," a symptom which in itself may constitute a complete morbid condition. At that time I referred to the fact that the majority of persons who show crystallised uric acid in their sediments are not sufferers from pollakiuria, but that in certain cases where changes exist in the neck of the bladder region or the prostatic urethra, themselves incapable of causing a pollakiuria, the paroxysmal excretion of large amounts of uric acid, especially in the form of pointed crystals, can produce a pollakiuria of high grade. Such paroxysms of uric acid excretion are not rarely seen in patients with arthritis, and if this excretion can be made to disappear by the administration of alkalies and other proper treatment, as, for instance, at Carlsbad, this distressing symptom will disappear as well.

No less a man than Sir Henry Thompson ${ }^{7}$ writes as follows concerning the effect of Carlsbad treatment on uric acid excretion :-

Now, the principle upon which the waters of the sulphate of soda group, aperient and non-aperient, are beneticial is, that they produce activity in all the digestive functions, stimulating the excretory action of the abdominal organs, so that certain waste matters, which have hitherto been thrown out as uric acid by the kidney, are eliminated in some other form. If, therefore, it is really desirable to recommend resort to a mineral spring as treatment for uric acid deposits. I should prefer Carlsbad, and certainly never send a patient to Vichy, Vals, Fvian, or Contrexèville. And when a stout active man, whom it is most desirable to separate from his home engagements and business advantage.

In our discussion thus far we have repeatedly touched upon the subject of cystitis. It is one of the most gratifying for thermal treatment. Under the influence of the drinking and bathing an acute, non-gonorrbœal cystitis usually recovers quickly without any local treatment. Those forms are especially often seen here which occur in connexion with gravel, apparently due to the insult to the mucous membrane from the uric acid concretions. Such a urine is, as is well known, acid, contains red and white blood corpuscles, pus cells, bladder epithelium in small quantities, and fewer or more of the pointed forms of uric acid crystals. Chronic cystitis with amphoteric or alkaline urine must of course be treated locally. But it is just here, and especially in the more stubborn forms, that the balneological treatment shows its value. I think it will be conceded that usually, in beginning local treatment, a physician cares relatively little for the systemic, that he confines himself to forbidding certain articles of food and drink (alcohol, spiced foods, \&c.), and is usually not in a position in the city to prescribe at the same time mineral drinks and therapentic baths. But it is most of all in connexion with chronic vesical catarrh that the value of this part of the treatment as adjuvant to local measures is under-estimated, as our practice testifies. Here the increase in metabolism performs great services-indeed, it often works miracles almost. Just what we imply by this increase in metabolism we have elsewhere discussed.

We must characterise as categorical and illogical that dogma which is too often voiced, "alkaline urine, ergo no alkalies, ergo no Carlsbad." The urine is alkaline in the "alkaline cystitis" because it contains pus and is in a state of ammoniacal fermentation. If this pus and fermentation be removed by local and general treatment the alkalinity of the urine disappears ipsn facto, and, paradoxical as this may seem, an alkaline cystitis never responds so well as under the influence of alkalies, especially those of the sulphate springs; these open all portals for the escape of the toxins of the colon bacillus and the rest. On the principle "when all else has failed, try Carlsbad" a great number of old protracted cases of cystitis are directed to Carlsbad. The people in question are the most grateful of our patients. In this class are those forms of old chronic cystitis which need constant or very protracted treatment, which are complicated with prostatic disease of high grade, strictures, \&c., and are accompanied by the remote symptoms of chronic uræmia. The sonde à demeure has been recommended for such cases to drain the bladder constantly. I must say that in general I am not an advocate of this method, for the permanent catheter requires the patient to stay in bed, and thus the already insufficient digestion suffers and the recumbent attitude favours hyperæmia of dependent parts, the prostate, the bladder, and the other pelvic organs The inactivity gives the metabolism and so the impulse to recovery another set-back. Here the open-air treatment shows to advantage and we may meet the indications for thorough bladder drainage just as well if we have the patient catheterised for every micturition--i.e., at least four times daily and once or twice at night-of course, with all aseptic precautions-and allow him enough outdoor exercise to stimulate the digestion and to meet the need for fresh air, a need that should not be underrated in such cases. In this way there can be no considerable stagnation of urine and the bladder is washed once or twice daily with boric solution (or with Müblbrunn or Sprudel water) and silver nitrate injected or instilled as well. ${ }^{8}$ The fresh-air treatment is important for these patients, as $I$ have already stated, so also are the baths, the massage, in some cases carefully selected light gymnastics, the régime, the stimulation to the digestion, and last, but not least, the flushing out of the urinary passages with sulphated alkaline waters. In the management of chronic diseases and of those of metabolism all possible curative agents must be called upon to enhance the effect of the drinking and bathing treatment.

We cannot refrain at this point from citing a remark by that keen medical thinker, $\overrightarrow{\mathbf{H}}$. A. Hoffmann. He says: "The great value of the Carlsbad treatment, which we are constantly prescribing, is so far from clear scientifically that one would lay himself open to the charge of phantastic theoreticism if one were to say anything definite on the subject." In fact, Carlsbad practice yields results which had better be taken thankfully and without racking the brains over the cur quo modo.

Hypertrophy of the prostate is seldom the subject of treatment unless it is associated with obesity, chronic arthritis, or especially with chronic constipation. In the first stage, when periodical feelings of pressure or fulness and the irritation in the rectum are the only complaints, a simple course of drinking and bathing, or of the former alone, will benefit without other adjuncts, without even prostatic massage. Here the case is usually one of paroxysmal congestion of the gland coming on under the influence of stasis in the lower pelvic organs. As soon as order is restored by regulating the digestion the complaints disappear. In such an aseptic case the introduction of an instrument should be avoided. On the other hand, where there is a large quantity of residual urine, where the impediment to the outflow is really considerable, one cannot too industriously drain, wash, and, as Guyon expressed it, " modify" the mucous membrane of the bladder. This is no place for a dissertation on prostatic hypertrophy, but we may say this: that the greatest effort should be made to prolong the first stage, or, in other words, to put a catheter into the patient's hands as late as possible. If the operation is indicated one must not wait until ascending processes have made the prognosis unfavourable. Such processes lead to the signs of chronic uræmia, which we have already fully discussed. All these patients catch cold easily, therefore especial caution must be observed; and since a "cold" is by no means a small matter for people with chronic urinary trouble they should be sent to a watering-place only in a favourable season. In the cold spring of 1905 I had the opportunity of seeing some particularly unpleasant instances of this. I shall always remember one case of an old gentleman with a tremendous enlargement of the stomach, with atony, an old cystitis, and prostatic hypertrophy. The treatment of the lastnamed was being postponed on account of the wretched general condition. After starting treatment the digestion improved, the stomach-tube, a necessity hitherto, was dispensed with, and the weight increased. Everything looked bright. Then the old man caught cold and the symptoms of acute prostatic congestion came on, with fever, with exacerbation of the cystitis, which, of course, worked back on the gastritis, producing vomiting, singultus, anorexia, and prostration. The clinical picture became so threatening that I felt myself fortunate when the treatment of the

8 The irrigations are best performed by means of the irrigator and use may well be made of a two-way tap. I demonstrated an aseptic model of such a tap in February, 1907, before the Gesellschait der Aerzte in Vienna. It may be obtained of $\mathbf{H}$. Reiner in Vienna. 9 Von Leyden: Handbuch der Frnährungstherapie, second edition,
1903, p. 481 . 
bladder, which I then had to begin, had after a few days removed the imminent danger. To such cases the experienced urologist is no stranger.

This brings us to a class of patients whom Guyon denominated as "faux urinaires," which could be translated as "psendo-urinary" cases, though the former, like many another French expression, has won its place in our urological nomenclature. ${ }^{10}$ Guyon understands by this term " patients with symptoms which are falsely localised by them, and which indicate a really existing disease, even if in another organ (i.e., other than the urinary apparatus)." We might go further and understand a, symptom-complex which can mislead not only the patient but the physician, and which may indeed imitate closely an affection of the urinary apparatus, thus leading to failure to supply appropriate treatment for the case. Perhaps one sees more of these in Carlsbad than anywhere else. To give an instance, the chronic dyspepsias with increased frequency of micturition are well known. I may mention here two cases of this type, such as are not particularly common.

A middle-aged gentleman, a professional brother, consulted me for a troublesome pollakiuria combined with pain in the urethra, and especially in the glans. Even the introduction of a Nélaton bougie caused extremely severe pain. The patient thought he had a stone and wished me to sound him. I declined, however, to make this examination, not only on account of the extreme sensitiveness of the urethra, but chiefly because the diagnosis was evident from symptoms. Eren such freely explored viae naturales as these should not, for mere curiosity, be explored with an instrument. Against my advice, however, the patient, to whom for sufficient reasons I had not told the real diagnosis, had a sound passed by another hand-of course with negative results, for before the examination was made I had told his family that he was suffering from those vesical and urethral crises which were described by Geffrier ${ }^{11}$ and Fournier ${ }^{12}$ as characteristic of the pre-ataxic stage of tabes dorsalis. In this case the drinking cure and hydriatric procedures had a favourable infuence on both the intestinal disturbances which were present and the course of the fundamental trouble. I have seen the patient lately and was astonished at finding how well he was doing.

This case is not exceptional ; tabetics have consulted me repeatedly in the earliest stages of their disease, on account of bladder disturbances, and I have always seen good results from the above-mentioned treatment. The good effects of hydriatric procedures in tabes are well known, but I wish to emphasise especially that by means of the drinking cure in addition we may meet the symptomatic indications and influence the intestinal and vesical disturbances.

The second of these "pseudo-urological" cases was the following. A patient was sent to me five years ago with the diagnosis of nephrolithiasis. As a matter of fact, he had passed a number of uratic calculi about a year before after a typical colicky attack. But at the time that I examined him, in spite of intermittent pain in the kidney region, there was neither sand nor gravel in the urine. The type of this pain failed, moreover, to simulate that of a renal colic closely enough to give me any real cause for doubt, and, in fact, on more careful examination I found it to be a case of sciatica and one which cleared up rapidly under the administration of alkalies, massage, and mud baths (it was apparently of the uratic form). The false diagnosis had been made, of course, on the basis of the former attack of renal colic and more particularly because the pain was chiefly in the distribution of the iliac branch of the iliohypogastric nerve-that is, about in the kidney region.

Similar psendo-renal colics may be imitated as well by chronic constipation, when deposits of hard scybala may be present in the splenic flexure, and more often by spastic constipation involving the descending colon. This frequently causes pain, often rather severe, which may radiate as far as to the right testicle, and is often increased by pressure over the splenic flexure. Appendicular and tubal colics may also be mentioned in this connexion.

There is another class of patients whom I should call " urinaires dissimules," or concealed urological cases. They form a considerable contingent of the visitors at Carlsbad. These are people who complain of little else than digestive

10 Guyon's Lectures, German edition, by Dr. Oscar Kraus and Dr. Otto Zuckerkandl, Vienna, 1894, vol. i., p. 18.

11 titude sur les Troubles de la Miction dans les Maladies du System Nerveux, Paris, 1884, Dissertation. 12 L'Ataxie Locomotrice d'Origine Syphilitique, 1882. disturbances of various kinds, of constipation, chronic diarrhoea, anorexia, hemicrania, \&c., symptoms which in reality are remote manifestations of a local affection of the urinary apparatus-i.e., of a chronic uræmia. In this character appear especially chronic nephritis and prostatic disease. These patients are in a particularly delicate equilibrium as regards health. They know that they "once had a catarrh of the bladder," but that was long ago, and they are used to it. They come to Carlsbad because of poor digestion. They have been advised to "give their bladder a complete rest for a while"-that is, not to have it treated locally! I have elsewhere reported one such case. ${ }^{13}$ But the physician is guilty of a serious sin of omission if he does not treat such cases locally and of a disastrously wrong diagnosis if he handles them as stomach or intestinal affections. I will cite here two cases.

The first case was that of a woman who was over 60 years of age and who had from time to time for many years come to Carlsbad on account of cholelithiasis. She had been for some time under treatment by different well-known gynæcologists for a pelvic trouble. She was referred to me by her own Carlsbad physician because she had "suddenly" begun to suffer from strangury. On examination I found a prolapse of the uterus and, as often happens in connexion with it, a prolapse of the anterior vaginal wall and a part of the bladder, so that this pocketlike portion did not empty itself-like the furrows in a prostatic case. In the same way there was here a residual urine amounting to between 50 and 100 cubic centimetres. A chronic cystitis was present which the patient considered of quite trifling importance. A well-known urologist, since then deceased, had directed her to catheterise herself over a hand-mirror and occasionally to irrigate the bladder. She had done so "occasionally" if the cystitis was troubling her. This time the strangury was annoying her unusually because as she said, she had "turned her stomach by eating bad peas." The bad peas here were a typical urinary fever, with profuse diarrhœea, almost unbearable tenesmus, nausea, \&c. Her condition caused considerable anxiety for 24 hours, but finally yielded to rest in bed, great quantities of hot sprudel water, and, for the pollakiuria, cocaine applications to the vulva. As soon as the patient could get up the cystitis was treated with success. The digestive complaints were not mentioned again. A pessary relieved the retention.

The second case was that of a woman from Northern Bohemia. She took to bed immediately on arrival at Carlsbad. She had been ailing for many months and was said to have biliary sand. On entering the room one could make a diagnosis of ammoniacal cystitis by the sense of smell. Also, in this case there was urinary fever; the bladder proved again to be the porta malorum, and appropriate local and general treatment resulted in a disappearance of the fever and an increase in the strength. This patient had also, as a matter of fact, an affection of the liver and was jaundiced; but it was impossible to refer all the symptoms, fever, prostration, anorexia, and diarrhea, to the biliary function. These were all remote symptoms of chronic uræmia. One must not be satisfied to refer all phenomena without afterthought to a disease that is known to exist, otherwise therapentical signs of omission may be committed with disastrous results.

I have elsewhere called attention to the fact that a pollakiuria in diabetes should always arouse suspicion of a nondiabetic cause. When such a patient complains of increased frequency of micturition one should not be satisfied to ascribe it merely to the diabetes and to consider everything thus explained, but should always look for a possible other reason. Almost without exception a complicating affection of the lower urinary passages may be demonstrated, which was the source of the pollakiuria. I am convinced that, although diabetes may cause a polyuria, it never, except in the early stages, and then not as often as supposed, causes a pollakiuria, or, as Clado, before his untimely death, more properly named it, a sychnuria.

Even though a polyuria does not as a rule cause a sychnuria, the bladder accommodating itself to the greater demands on its capacity, the converse may well happen that sychnuria can cause a polyuria. Our experience leads us to agree fully with Guyon to the effect that an increase in the frequency of emptying the bladder reflexly increases the kidney secretion and conversely that the decrease or total

13 Congress of the Association Française d'Urologie, 1897. 
relief of this abnormal frequency decreases in consequence the quantity of urine.

Of the many examples which could be given to support the above statements I mention but one. This was the case of a Parisian woman who was under our care in September, 1901. She suffered from severe diabetes, discovered in 1895, and had been treated since then with indifferent success. When we first saw the patient the excretion of sugar, in spite of strict diet, amounted to 48 grammes per litre, with a daily quantity of urine of over 3000 cubic centimetres, or a total excretion of about 150 grammes of sugar. In addition there was abundant excretion of acetone and diacetic acid and a trace of $\beta$-oxybutyric acid. The specific gravity was 1030, with an ammoniacal reaction, while in the sediment were found mucus, pus, and triple-phosphate crystals. The cystitis was a matter of years' standing and was the cause of an unusual increase in the frequency of micturition; by day the patient had to urinate about every half hour and averaged six or eight times at night; and all of this had been ascribed to the diabetes! As for other symptoms it need only be mentioned that the patient had suffered for years from chronic constipation and had lately lost much weight. I began local treatment of the bladder; the urine soon became clear and acid, with simultaneous disappearance of the sychnuria, although the polyuria still continued, and the sugar decreased but slowly, as a diet free from carbodydrates could not be introduced at once on account of the acetone. It was only much later that the sugar and the polyuria decreased together (daily quantity 650 cubic centimetres) and the patient gained weight.

As a résumé it may be said that the Carlsbad waters are particularly indicated in diseases of the urinary passages for the following reasons. 1. Because they are notably diuretic. Therefore they have an eliminative action on foreign bodies in the urinary passages, like clots, mucus, pus, or small concretions, such as can be passed out per vias naturales. The passages are "well tlushed out." Of course, micro-organisms (bacillus coli, \&c.) are also by this means washed out. 2. They have a modifying action on the urinary mucous membrane ; catarrhs of low grade heal rapidly without local treatment. 3. The Carlsbad waters stimulate both renal and intestinal functions energetically and their use is thus especially indicated in those cases where so-called urotoxic substances are to be eliminated-i.e., in cases of prostatic disease, chronic cystitis, \&c.- -and in those cases of nephritis where the gastric symptoms prevail. 4. The regulation of intestinal activity has a direct depleting effect on the pelvic organs, bladder, prostate, \&c. The venous stasis due to intestinal stagnation is mitigated, the deeper organs are relieved. The increase in volume of the hypertrophied prostate due not to secondary degenerative changes but to a congestive hyperæmia-and in every case a part is congestive-disappears as a result of the regulation of the bowels. 5. Post-operation, after nephrotomy, suprapubic lithotomy, litholapaxy: to wash out the smallest particles of sand; after prostatectomy : to alleviate the cystitis, regulate the bowels, and eliminate the toxins. 6. The Carlsbad treatment is contra-indicated in the common condition of frank urogenital tuberculosis, so frequently taken for a simple cystitis or pyelitis.

Carlsbad.

\section{atteoural Socretics.}

\section{CLINICAL SOCIETY OF LONDON.}

\section{Exhibition of Cases,}

A MEETING of this society was held on April 26th, Mr. H. H. Clutton, the President, being in the chair.

Mr. LAWRIE H. MCGAVIN exhibited two cases of Ventral Hernia through an operation-scar treated by the Implantation of Large Filigrees of Silver Wire. The first case, that of a woman, aged 38 years, presented a hernia at the site of the scar of an operation that had been done a year before, the gap measuring 5 inches by 3 inches. The whole of the old cicatrix was removed by an elliptical incision 11 inches in length. One portion of the external oblique aponeurosis was sutured to the cut edge of the posterior sheath of the rectus muscle and upon this, as a bed, a silver wire filigree, measuring 8 inches by 4 inches, was laid, the inner loops being disposed beneath the rectus muscle and the onter beneath the oblique muscles. The remains of the rectus muscle were now brought out of the sheath and sutured as far as possible to the oblique muscles over the filigree. This caused a weak spot at the middle line of the abdomen and necessitated the opening of the sheath of the left rectus muscle, which was drawn across the middle line to meet its fellow, where it was sutured. The skin and superficial fascia were closed over all by 35 Michel's clips. No belt had been worn since convalescence. The second case presented a similar condition after an operation for appendicitis. The scar had been treated in like manner and the result had been most satisfactory.-Mr. A. E. J. BARKeR congratulated Mr. McGavin on his results. The method was certainly superior to any he himself had used and saved a great deal of time and manipulation. He had used interlacing wire in about 30 cases and in only two instances had it to be removed subsequently. He referred to the respiratory embarrassment which he had seen occur after the replacement of enormous hernix. - Mr. McGavin said in reply that he had not had to remove the filigree in any of the 16 cases he had done.

Mr. MCGAviN also showed a case of Inguino-scrotal Hernia in a male treated by Implantation of Filigrees of Silver Wire.

Mr. DoUgLas DREW exhibited two cases illustrating the Use of a Filigree of Silver Wire in the Radical Cure of Umbilical Hernia.

Mr. DREW also showed a case of Habitual Dislo cattion of the Patella (? Congenital) in a girl, aged 13 years. The child first came under observation when six years old with severe genu valgum and dislocation of the patella when the knee was flexed. Macewen's osteotomy of the femur was performed in April, 1900. The present condition was much the same as seven years ago. On flexing the knee the patella slipped completely over to the outer surface of the external condyle, whereas when the knee was extended it lay over the external condyle. It was suggested to treat the case by dividing the capsule on the outer side of the patella so as to allow of the reposition of the bone, and by shortening up the capsule on the inner side and deepen. ing the patellar surface on the femur.-Mr. WALTER G. SPENCER advised the removal of the patella, care being taken not to damage the aponeurosis.-Mr. DREW recalled a case operated on in the way which he now suggested by Mr. Bilton Pollard 16 years ago, with an excellent result.

Dr. F. E. BATten showed three cases of Acute Ataxia (Encephalitis Cerebelli). The first case, in a boy, now aged six years, had been exhibited before the society in October, 1904. In April, 1904, he vomited, had general convulsions, and passed into a comatose condition. On regaining consciousness he was wildly ataxic alike as regards his legs arms, and trunk. From this he very slowly recovered and when exhibited before the society in 1904 was still very ataxic. He walked fairly well but was still unsteady, especially on the left leg. The articulation was still slow and hesitating. The reflexes were normal. Sensation to touch and pain was perfect. The second case was in a boy, aged 11 years, who was quite well up to April 1st. He had severe headache, vomited a whole day, and was unable to feed himself or to stand. On April 5th he tried to get out of bed and walked like a drunken man. On the 9th he had marked ataxia of both legs, some ataxia of the hand, and nystagmus on looking to the left side. There was loss of tone in the muscles of the lower limbs. The knee-jerks were active and the plantar reflexes flexor. The boy had rapidly improved, so that he now only exhibited slight ataxia in the lower extremities. The third case was that of a boy, aged 12 years, who had always been backward. For the last seven years the patient had had attacks of petit mal. In September, 1906, he had a series of 130 fits and was unconscious for three days. On recovery he could move his arms and legs but had no control over them and had not been able to walk since. His mental condition was poor and his articulation was slow and jerky. There was unsteadiness of the trunk and head. There was general weakness of the arms and a well-marked intention tremor was present. He could not walk without aid and his gait was wildly ataric. Dr. Batten referred to the favourable prospect in these cases and recalled Dr. Frederick 'Taylor's case that made only slight improvement in the first three years but had ferfectly recovered when seen 25 years later.

Mr. O. GoRdon Watson showed two cases of Modified Pirogoff's Amputation in men, aged 30 and 40 years re. spectively. The operation was performed in the first case for 